\title{
Cryoprotectants and Their Usage in Cryopreservation Process
}

\author{
Sankha Bhattacharya \\ Additional information is available at the end of the chapter \\ http://dx.doi.org/10.5772/intechopen.80477
}

\begin{abstract}
Cryoprotectants are basically some chemical compounds which prevent cells or tissues from damage due to freezing. Mostly vitrification and thawing process are mostly used in cryopreservation. Now a day's cryopreservation is becoming an apex technology for Health sector, as many organ and tissue transplant methods has been developed in recent time. Organs or tissues need to preserve properly before implantation, hence cryoprotectants plays a key role modern cryonics. This article seeks to provide answers for how cryoprotectants works and prevent tissue damage from shrinking ice crystals during freeze drying or vitrification. The various animal tissues, organs, lymphocytes, cartilages, bone marrow, proteins can be stored by using proper cryoprotectants. Optimization of cryoprotectants is a challenging task, as elevated concentration may cause cytotoxicity. This article also highlights the implication, challenges and recent advances of cryoprotectants along with its types. The importance of cryobank system and its importance were also being emphasized in this article. Further, importance of cryoprotectants in nano suspension preparation was also been discussed in this article.
\end{abstract}

Keywords: cryopreservation, vitrification, cryoprotectants, cryobank system, dimethyl sulfoxide, glycerol

\section{Introduction}

To prevent cell death during preservation, tissues and organs temperature used to lower to stop cell death. But at the same time rate of cooling also affect the cell survival. The preserving cells which are cools quickly are susceptible for cell death due to rapid intracellular ice formation. Sometimes due to dehydration, cells can die. In other way, due to the presence of hypotonic solutions surrounding of cells can also leads to cell shrinking and cell death. 
The deleterious affect associated with slow drying was sorted out by using glycerol as cryoprotectant during 1949. Without proper cryoprotectant cells ruptures due to "Solution effect" injury; in which residual solution of cells altars shape, mechanical strength of the cells followed by osmatic oscillation during freeze drying. What basically cryoprotectants do is after dissolving in preserving solution it lowers the melting point of the solution so that tissue or organs can freeze within its surroundings but not within its cells [1]. These cryoprotectants are sometime called as antifreeze. There are so many cryoprotectants but glycerol, propylene glycol, dimethyl sulfoxide (DMSO) are most commonly used within this category. For the cell survival after freezing and thawing of liquid nitrogen temperature, it became necessary to preserve tissues with suitable cryoprotectant (5-15\%) which helps to form large unfreeze pockets [2]. These pockets helps to prevent crystal formation and mechanical damage. During freeze drying process organs and tissues are venerable to freezing injury, for any organ to reestablished function after freeze drying has to have intact parenchymal cell to cell of small blood vessels. Only $25 \%$ of cell survival during freeze drying is not enough, hence a new approach of tissue preservation called vitrification is required to protect tissues. Vitrification means turns into a glass. Fathy, advocated vitrification, in which tissue or organ encircled into unfrozen glassy pocket containing cryoprotectant, it was planned, tissues to be treated with maximum cryoprotectant before freeze drying to avoid ice formation [3]. Due to the rapid acceptance of verification and slow freezing process, a maximum number of animal germ plasmas, tissues were started to store by these process. Further advancement embarks the idea to preserve tissues below -130 to $-196^{\circ} \mathrm{C}$, which is basically a sub-zero temperature using some substances. It was also observed that all the associated problems of refrigerating storage such as integrity of living cells while storing, freezing injury, forming ice crystals which eventually damage stored cells, could be restricted from using such substance. These substances are also helping in moisturizing the living cells by penetrating inside of stored cells without altering or creating any lethal effect to those cells, such substances are called as the cryoprotectants or the cryopreservatives. Without cryoprotectants at low-temperature biological cells damaged, and at in transition phase such as changes from frozen solid to a liquid by a general warming, cells loses numbness and stiffness, this condition is called thawing. Mostly slow freezing is preferable for preserving cells in which cells are frizzed slowly below its freezing point, but at a certain point, the formation of spherical crystals can destroy cells chronobiology. These crystals are called as unfrozen fractions [5]. Using cryoprotectants along with some electrolytes and sugars it is possible to increase osmatic strength of the solvent by which positive efflux of moisture towards the cells occur and during this process chances of intercellular ice, the formation becomes negligible. Farther cooling causes increase of viscosity and due to this, chances of formations of ice crystals would become very infinitesimal. In the last stage of slow cooling, remaining unfrozen fraction transformed to amorphous solids without ice crystals. The main objective of cryopreservation is to create a dehydrating environment for preserving cells. In this process cytosol observed less injury during cold freezing within liquid nitrogen, as numbers of crystalline substances formation would be very minimal. Mostly it was found that cryoinjuries occur during anomaly of instantaneous super freezing of cells. Slow freezing's with lucid cryoprotectants causes fewer injuries [6]. Eventually, cryoinjuries occurs during pre-freezing and post-thawing, within the temperature of $0-40^{\circ} \mathrm{C}$. During cryopreservation technique, $\mathrm{pH}$, osmotic pressure, and concentration 
of cryoprotectant has to be maintained, as elevation can cause cytoinjuries. In some cases, if the cell cytosol contains specific fatty acids, i.e., spermatozoa plasma membrane, it helps to maintain proper osmotic pressure and resist shock during thawing and freezing, by which fatty acids helps to maintain cells rehydration.

\section{Toxicity due to cryoprotectant}

During cryopreservation process using vitrification or freezing, almost half of the cell fluids were replaced with cryoprotectant molecules. These cryoprotectant molecules some time cause toxicity while in warm temperature. For example as a cryoprotectant in warm condition, propylene glycol is nontoxic while ethylene glycol metabolized to toxic elements. Recently it was found that lipophilicity of cryoprotectant could help in deeper penetration of cryoprotectant into cells causes destabilization of cells. On the other hand, strong hydrogen bonding also correlates with toxicity by disrupting the hydration shell around macromolecules. The electrical properties of cryoprotectant solution also has membrane toxicity. Among all cryoprotectants, DMSO is severely toxic, basically DMSO/formamide solution has been useful in vitrification process, but mechanism of biomedical toxicity reduction is still unknown $[1,4]$.

\section{Various components of cryoprotectant solution}

Cryoprotectants solution may be a freezing solution or sometimes it may be a carrier solution. The carrier solution should not be an explicit cryoprotectant. Carrier solution helps to provide minimum support for cells at freezing temperature. Carrier solution often comprise of nutritional salts, buffers, osmogens, and apoptosis inhibitors. Often ingredients maintains isotonic concentration (300 milliosmoles) so that cells neither swells nor shrink when held in carrier solutions. These carrier solutions sometimes called as "base perfusate" solution. Carrier solution consisting of M22 cryoprotectants is called as LM5. During the process of freezing the carrier solution concentration always remains constant. Like carrier cryoprotectants there is another type of cryoprotectants called penetrating cryoprotectants, the basic role of penetrating cryoprotectants is to reduce cell dehydration and ice growth. The penetrating cryoprotectants are the major ingredients of vitrification solutions. Certain large molecular polymers are also been added to cryoprotectant solutions, they literally inhibits ice growth using same mechanism of penetrating cryoprotectants, these are called as Nonpenetrating cryoprotectants. Polyvinylpyrrolidone (PVP) and polyethylene glycol (PEG) are examples of non-penetrating cryoprotectants. These cryoprotectants are highly toxic as compare to penetrating cryoprotectants in same concentration. Recently it was discovered that non-penetrating cryoprotectants can increase tonicity of vitrification solutions, which helps to prevent chilling injury.

Some ingredients in cryoprotectants solution called ice blockers, which are helping directly to block ice growth. Some examples are polyglycerol, polyvinyl alcohol, X-1000 and Z-1000. Ice blockers are used only in vitrification solution [4, 6-8]. 


\subsection{Utilization of cryoprotectants}

During cryopreservation, initially nearly $10 \%$ cryoprotectants were added in a single step. This causes classic shrink swell response of cryobiology, in which cells first shrink due to osmosis and later swell by penetrating cryoprotectants enters the cell. Unless and until cryoprotectants volumes equals between intra cellular and extra cellular fluids of cells swelling persist. Within $10 \mathrm{~min}$ of concentration and volume adjustment, cell or tissue is ready for freezing. The cooling process is done very slowly, mostly less than $1^{\circ} \mathrm{C} / \mathrm{min}$. The addition of vitrification solutions during cryopreservation should not be exist $50 \%$ in a single steps, because of elevated osmatic shrinking response. The material's which need to be vitrified exposed to several cryoprotectant solutions such as $1 / 8 \times, 1 / 4 \times, 1 / 2 \times, 1 \times$ full concentration vitrification solution as specially for $20 \mathrm{~min}$ each steps. During vitrification cooling and rewarming are done rapidly. During this process cryoprotectant solutions just perfused through cells like blood flow in blood vessels. After vitrification, cryoprotectant can be remove by reversing the steps describe earlier [9-13].

\section{Mechanism of cryopreservation}

The melting point of water decreases after proper mixing with suitable cryoprotectant. The various anti-freezing cryoprotectants are dimethyl sulfoxide (DMSO), propylene glycol. During freezing and thawing of isolated cells mostly $5-15 \%$ concentration of cryoprotectant concentration has to be maintained for better result. With cryoprotectant the freezing of cells in liquid nitrogen would be slower, further formation of relatively large vesicles and fewer salt crystals helps to protect cells from any mechanical injuries. As per evidence cryoprotectants usually do not forms any hydrates and they are relatively less toxic to cells in elevated concentration. Vast expensive use of glycerol as cryoprotectants becomes a stepping stone towards finding more than hundred more cryoprotectants within 50 years. In new age penetrating cryoprotectants were using vastly in the pharmaceutical industry because they are having a molecular mass of fewer than $100 \mathrm{Da}$ and they penetrate inside of the cells and maintain moisture during freeze drying [4, 9].

\section{Some common cryoprotectants used in pharmaceutical preservation}

\subsection{Dimethyl sulfoxide (DMSO)}

The Great Russian scientist Alexander Zaytsevin on 1866 synthesized DMSO [4]. Basically DMSO is an organosulfur compound with the formula $\left(\mathrm{CH}_{3}\right)_{2} \mathrm{SO}$. It is also a polar aprotic solvent which can dissolve polar and nonpolar compounds and can be easily miscible with wide range of organic solvents and with water. DMSO has low cost and minor cytotoxicity, which makes it more prominent candidate for cryopreservation. It has garlic like taste. At any particular temperature, DMSO reduces the electrolytic concentration in the residual chilled 
contents in and around of a biological cell, during cryopreservation. However rising of altered demarcated cells due to DNA methylation and histone alteration is a drawback of DMSO based cryopreservation. DMSO has typical property; it freezes within $18.5^{\circ} \mathrm{C}$. This means, below room temperature DMSO transformed into solids, and this property makes it most suitable for cryoprotectant.

\subsection{Ethylene glycol}

Voelkel et al. [27] used 1.5 M ethylene glycol as a cryoprotectant for bovine embryols allowing direct transfer of frozen-thawed embryos to recipient females. Ethylene glycol alters the hydrogen bonding when it mix with water. Purified ethylene glycol has a freezing point at about $-12^{\circ} \mathrm{C}$, but after mixing with $40 \%$ water and $60 \%$ ethylene glycol the freezing point of the mixture would depressed and mixture becomes incapable of forming crystalline substances. This condition leads to transformed freezing point at $-45^{\circ} \mathrm{C}$. This property of ethylene glycol makes it the most effective candidate for cryoprotection. But some toxicity was observed for ethylene glycol, like GI irritation, pulmonary edema, and lung inflammation.

\subsection{Glycerol}

Oana Lelia Pop et al. [28] studied the effect of glycerol as cryoprotectant during freeze drying of microspheres containing probiotic cells. The colorless, the odorless viscous liquid of simple polyol (sugar alcohol) compound named as glycerol or glycerine. Glycerol has good kosmotropic properties; it forms hydrogen bonds with water molecules. This condition makes difficult to form ice crystals by mixture (70\% glycerol and 30\% water), unless and until the temperature is very low such as $-37.8^{\circ} \mathrm{C}$. Compare to other cryoprotectant glycerol is less toxic in high concentration.

\subsection{Propylene glycol}

Propylene glycol IUPAC name is propane-1, 2-diol. It is basically a non-irritating synthetic organic compound with a chemical formula $\mathrm{C}_{3} \mathrm{H}_{8} \mathrm{O}_{2}$. It possess a fairly sweet taste with color less and odorless characteristics. Chemically it is a diol and miscible with water, chloroform, acetone. It is widely used as aircraft deicing fluid. Propylene glycol is sold under the name of $\mathrm{RV}$ or marine antifreeze. It also carries the property of automotive antifreeze.

\subsection{2-methyl-2, 4-pentanediol (MPD)}

MPD widely used as precipitant, but in protein crystallography, it can be used as a cryoprotectant. It can be vividly used with polar and non-polar solvent. It can help the protein to precipitate.

\subsection{Trehalose}

This compound comprising with two molecules of glucose. Trehalose is otherwise called as mycose or tremalose. Due to its high water retaining properties it can be used as cryoprotectant. 
Trehalose is less soluble than sucrose, except at high temperatures $\left(>80^{\circ} \mathrm{C}\right)$. Trehalose forms rhomboid crystals of $90 \%$ calorific sucrose. The anhydrous forms of trehalose readily regain moisture to form the dehydrate. Trehalose improves cell survival after thawing compared with the standard freezing procedure. Sometimes trehalose can be used along with hyaluronic acid to treat dry eye.

\subsection{The cell bank series}

The cell banker series containing cryoprotectants media has 10\% DMSO, a specific polymer, $\mathrm{pH}$ modifiers, glucose, bovine serum albumin. This combined cryopreservatives are used to preserve mammalian cells. This techniques allows rapid cell cryopreservation at $-80^{\circ} \mathrm{C}$ and mean survival rate of cells after freezing and thawing was outstanding.

\subsection{Formamide}

It is also known as methanamide. It is derived from formic acid. It has ammonia like odder and clearly miscible with water. As a cryoprotectant, it helps to soften tissue vessels. It is also been used as a resins and plasticizer. The most common formamide is called as dimethylformamide, $\left(\mathrm{CH}_{3}\right)_{2} \mathrm{NCHO}$.

\subsection{Glycerol 3-phosphate}

This chemical compound comprising of glycerophospholipids. This product is also called as 3-O-phosphonoglycerol.

\subsection{Proline}

Proline catalyzes aldol condensation. L-Proline is an osmoprotectant and therefore it has versatile use in Pharmaceutical and Biotechnological applications. Proline is actively participating in biosynthesis of protein. Proline has $\alpha$-amino group, a $\alpha$-carboxylic group, pyrrolidine side chain, aliphatic amino acid group.

\subsection{Sorbitol}

Sorbitol is otherwise called as glucitol. The best part of sorbitol is, its get metabolize in tissue very slowly. During reduction of glucose aldehyde group reduced to hydroxyl group. Mostly sorbitol is prepared from corn syrup. Basically sorbitol is an isomer of mannitol. Apart from good cryoprotectant property sorbitol also used for hyperkalemia.

\subsection{Diethyl glycol}

It is an organic compound with a formula $\left(\mathrm{HOCH}_{2} \mathrm{CH}_{2}\right)_{2} \mathrm{O}$. It is colorless and odorless poisonous hygroscopic compound. It is miscible with water, alcohol, acetone and ethylene glycol. Diethyl glycol is produce by partial hydrolysis of ethylene oxide. The dilute solution of diethyl glycol is a good cryoprotectant. A dilute solution of diethylene glycol can also be used 
as a cryoprotectant; however, ethylene glycol is much more commonly used. Most ethylene glycol antifreeze contains a few percent diethylene glycol, present as a byproduct of ethylene glycol production.

\subsection{Sucrose}

Sucrose is actually naturally occurring carbohydrates sucrose in low temperature $\left(-45^{\circ} \mathrm{C}\right)$, provides required nutrition to preserved cells, sucrose with the combination of DMSO maintains good cyroprotection properties.

\subsection{Triethylene glycol}

It is a colorless odorless viscous liquid with a molecular formula as $\mathrm{HOCH}_{2} \mathrm{CH}_{2} \mathrm{OCH}_{2} \mathrm{CH}_{2} \mathrm{OCH}_{2}$ $\mathrm{CH}_{2} \mathrm{O}$.

\subsection{Polymers}

It is possible to trauma biological materials using selective cryoprotective agents. Among all encapsulating materials, non-diffusible synthetic polymers can provide ridge cryoprotections of the biological cells within. For example polyvinyl alcohol, PEG, hydroxyethyl starch has good tendency to decrease the size of ice crystals.

\section{Type of cryopreservation}

\subsection{Isochoric cryopreservation}

Mostly all cryopreservation is based on an isobaric (constant pressure) process where freezing occurs at $1 \mathrm{~atm}$ pressure. But it has its own disadvantages, in this process intercellular ionic concentration increase, due to which chemical integrity changes within the cells during freezing, which causes cell damage. But in the isochoric (constant volume) process the metabolic rate of frizzing constantly changing with every 10 degrees of temperature reduction. In the isochoric process, it is possible to store at absolute zero temperature and it helps to maintain cell integrity during slow frizzing [14].

\subsection{Isobaric cryopreservation}

Isobaric cryopreservation technique deals with the preservation of cells in one atmospheric pressure. This process is widely used, but the certain limitation of cyto cellular damage makes it more bizarre.

\subsection{Hyperbaric cryopreservation}

This cryopreservation method maintains low temperature $\left(0^{\circ} \mathrm{C}\right)$ and high pressure followed by gradual demotion of pressure which causes rapid freezing. Hyperbaric preservation 
allows cells to be maintained at a low temperature without freezing and in an aqueous phase with one study reporting that survival was a function of both compression rate and final pressure magnitude. Despite the deleterious effect of pressure maintenance, some tissues and cells shown good preservation. For example red blood cells survived pressure up to $200 \mathrm{MPa}$, where else liver up to $35 \mathrm{MPa}$. By increasing elevated pressure it reduces temperature to sub-zero Celsius [15-17]. This condition leads to rapid freezing of biological tissues, by which many tissues can be stored by this process, i.e., kidney (10,000 atm), cells (200 atm), and liver (70 atm).

\section{Optimizing freezing}

Freezing or cooling optimization is a big issue in cryopreservation. Optimizing freezing condition with cryoprotectant needs $3-4 \mathrm{~h}$ interventions. The slow crystal formation process called seeding can damage cellular integrity. Optimizing freezing rate and concentration of cryoprotectant are very important for long storage of cells.

If cells were cooled very slowly and steadily, then extracellular fluids of the cells form ice crystals which pinch the cells to withdraw all intracellular component.

At elevated $-350^{\circ} \mathrm{C}$ temperature cells can lost its structure and shape. On the other hand, rapid cooling can cause serious damage to intracellular integrate of cells, where cytosol, parenchyma, nucleus and almost all cellular component rapidly freeze, which causes intracellular formation. All the integral parts of cells may come out and cell may die due to shrinking [18-20].

Hence, it is important to optimize cryoprotectant concentration with cooling rate. The optimize cooling requires the optimum intake of cryoprotectant within the cells and maintaining the integrity of the cells by not forming ice crystals while freezing, for example, human oocytes and embryos need minimum $90 \mathrm{~min}$ for preservation. Certain limitation of slow cooling makes it more challenging, such as it takes more sophisticated instruments with more time for cooling and at the same time certain tissues such as in vitro derived bovine, pig embryos, human MII oocytes, and blastocysts are very susceptible to chilling injuries.

\subsection{Carrier solution or base perfusate used in cryoprotection}

The carrier solutions acts as a life support or buffering substance for preserving cell. The main purpose of carrier cell is to maintain life integrity in cells during near freezing condition. Carrier solutions constituted with some essential buffer, osmogens, nutritional elements, salts and certain apoptosis inhibitors. To maintain stability within the cells, carrier solution maintains iso-osmolarity, which makes cells not to lose its original shape. For example M22, cryoprotectant solution is used with specific carrier solution is called as LM5. Most importantly carrier solution concentration cannot be altered with altering cryoprotectant concentration. It should remain constant, based upon the cells to be preserve. 


\subsubsection{Ice blockers}

Ice blockers are the substance which prevents ice to grow. While temperature is low and pressure is high this ice blocker binds with contaminants or ice inculcator to cleave ice formation. Examples are low molecular weight polyvinyl alcohol, polyglycerol, called as X-1000 and Z-1000. Mostly ice blocker is used in vitrification process.

\subsection{Cryopreservation using nanosuspension}

Nae-Oh Chung et al. [29], had tried to give an in-depth understanding of aggregation process of nanoparticles freeze-drying technique, they also outlines the importance of Cryoprotectants during lyophilization process. Yancai Wang et al. [30] had tried to give an outline of choosing cryoprotectant in freeze dried nanosuspension with suitable functional stabilizers. Cryopreservation and freeze drying both are very efficient ways to improve long term stability of nanosuspension. In Yancai Wang et al. work, mannitol (5\% w/v), trehalose $(5 \% \mathrm{w} / \mathrm{w})$, lactose $(7 \% \mathrm{w} / \mathrm{v})$, sucrose $(10 \% \mathrm{w} / \mathrm{v})$ was considered as an optimized cryoprotectants for preparing resveratrol (RSV) and quercetin (QUE) nano suspension. During this process of preparing Nano suspension using anti-solvent precipitation method, D- $\alpha$-tocopheryl polyethylene glycol succinate (TPGS) and folate modified distearoylphosphatidyl ethanolamine-polyethylene glycol (DSPE-PEG-FA) was used as a functional stabilizers. Upon storage for 90 days, it was observed that RSV \& QUE nano suspension containing mannitol 5\% w/v has limited deviation in particle size, enhanced dissolution rate, good physical stability as compare with other optimized cryoprotectants containing RSV \& QUE nano suspensions.

\section{Recent advances in cryopreservation}

Due to recent advancement of organ and tissue transplantation, it is important to have cryoprotectants research. Roger Gosden [31] did some extensive research on preserving ovarian tissues. He used 0.1 M fructose and dimethyl sulfoxide (DMSO) to preserve seven rat ovaries. It takes over $30 \mathrm{~min}$ to freeze and stored in liquid nitrogen overnight before thawing. Arav et al. [19] develops a novel technique using 1.4 M DMSO (10\% by volume) for preserving ovaries. This device transplanted in a test tube where freezing of organs can be possible between 0 and $-35^{\circ} \mathrm{C}$. This device far end is super cooled and stable. The organs which is about to preserve at first must be freeze in liquid nitrogen than exposed and thawed to $68^{\circ} \mathrm{C}$ for $20 \mathrm{~s}$ than $37^{\circ} \mathrm{C}$ for $2 \mathrm{~min}$. In this process preserved organ shown immediate blood perfusion after transplantation. Dittrich et al. [20] developed a method by freezing pig uteri in near dry ice temperature. It was tried to prove that this process is most efficient then vitrification technique. Viatcheslav Berejnov et al. [21] studied the effect of cooling rate on vitrification of aqueous solution. It was observed that $\mathrm{T}=295 \mathrm{~K}$ to $\mathrm{T}=77 \mathrm{~K}$ temperature, vitrification is reported in liquid nitrogen for almost all the cryopreservatives. It was concluded and confirmed that by X-ray crystallography, the transition from polycrystalline to vitreous occurs with in the span of $2 \% \mathrm{w} / \mathrm{v}$ in cryopreservatives. By which polycrystalline ice from hexagonal to cubic depends upon the elevation of cryopreservatives cooling rate and concentration. Anderson et al. [22] 
studied about ovarian cryopreservation for fertility preservation. They concluded that oocyte cryopreservation technique has more advantages as it produces approximately 100 fold more babies as it can preserve large number of oocytes within primordial follicles without further hormonal therapy. Chong et al. [23] studied about cryopreservation of neurospheres which is derived from human glioblastoma multiforme. Cryopreservation of neurospheres cried out by using $90 \%$ serum and $10 \%$ dimethyl sulfoxide. Further vitrification yields self-renewal and multi potential properties. Xu et al. (2010) studied about the role of apoptotic pathway in the low recovery rate after cryopreservation of dissociated human embryonic stem cells. It was observed that recovery of cells after cryopreservation of human embryonic stem cells (hES) become a huge challenge. It was found that the generation of reactive oxygen species (ROS) is significantly increased with F-actin altered distribution. Further analysis shown the activation of and caspase- 8 and caspase- 9 , which causes increase in cellular toxicity. Cetinkaya and Arat [24] studied about bio-banking system of cryopreservation for cartilage and tissue cells. Vitrification is used to preserve primary adult cartilage and fatal cartilage cells. The cartilage cells were slowly freeze using ethylene glycol (EG), Ficoll and sucrose. During slow cooling three different cooling rate were set as $0.5,1$ and $2{ }^{\circ} \mathrm{C} / \mathrm{min}$. Results astonished all by no significant changes in viability rations, proliferative activity and GAG synthesis observed after vitrification $\left(1^{\circ} \mathrm{C} / \mathrm{min}\right)$. This study emphasis the needs of vitrification in bio-banking of cell donor sources in nuclear transfer studies. Wong et al. [25] did vital studies on human embryos cryopreservation and its fertilization rate. In modern days cyroprotection human embryos cells is routine work, but optimizing success rate of transplantation is a matter of question. In this study high quality randomized trials were used to find best cryoprotectant protocol for fresh transfer of embryos. Silva et al. [26] reviewed about mammalian conservation biology and cryopreservation. They enlightened the importance of germ plasma cryopreservation of embryos, gonadal tissues, gametes, and some somatic tissues. They also dignified the importance of more usage of cryobanking for the preservation of cells and tissues of threatened species.

\subsection{Application of cryopreservation}

Now a days cryoprotectants has some versatile use. Mostly in preserving organ cells, in molecular biology, cryosurgery, blood transfusion, artificial insemination, bone marrow transplantation, in-vitro fertilization and most recently in identifying unknown transmissible disease or pathogen. During 1996, first embryo preservation for fertility was took place using IVF cycle. IVF cycle was prerequisite before chemotherapy of breast cancer diagnosed patients. Preserving oocytes using cryoprotectants is already an established technique. This technique causes no potential side effects on parturition. Same way, sperm and semen can be used after proper cryopreservation. Preserving testicular gonads using cryopreservation is still in infancy, but in future cryopreservation can be used in vasectomy.

\subsection{Limitation of cryopreservation}

In cryopreservation technique, at $-196^{\circ} \mathrm{C}$ in liquid nitrogen cells stops metabolizing which leads to unavoidable side effects. Sometimes there will be slow genetic changes occurs within 
the biological cells associated with the lipids and proteins, that could disfigure integrity of the cells. Often it was witnessed that, cryoprotective agents could damage chromosome stability of cells, like in higher concentration DMSO could produce tumor and also some times cryoprotectants makes cells susceptible towards infections.

\section{Conclusion}

Cryobiology will have a significant impotence in near future as a number of transplantation of vital organs of humans are becoming more demanding in the modern age. Yet, so many important complications are to be mitigated, like the exact mechanism of cryoprotectant, mode of action and cellular interactions with potential toxicity. More futuristic research is needed in respect of optimizing cryoprotectant concentration and slow and high freezing process. It was observed that macromolecular cells can easily preserve by cryoprotectant but as far as large tissues or organs (kidney, liver, heart, lungs, and skin tissues) are concerned, the success rate of freezing is not up to the mark by using cryoprotectant. Further optimized research on selection of specific concentration of cryoprotectants, freezing and thawing rates, best infusibility, and equilibrium times in cryopreservation might lead to better cell survival during preservation. Modern emerging cryobank is a promising approach towards organ transplantation. But still, standardization and optimization techniques are warrant for best cryopreservation.

\section{Author details}

Sankha Bhattacharya

Address all correspondence to: sankhabhatt@gmail.com

Department of Pharmaceutics, ROFEL, Shri. G. M. Bilakhia College of Pharmacy, Gujarat, India

\section{References}

[1] Polge C, Smith AU, Parks AS. Revival of spermatozoa after vitrification and dehydration at low temperature. Nature. 1949;164:666

[2] Wowk B, Leitl E, Rasch CM, Mesbah-Karimi N, Harris SB, Fahy GM. Vitrification enhancement by synthetic ice blocking agents. Cryobiology. 2000;40(3):228-236

[3] Pegg DE. Cryobiology. In: Proceedings of the Fourth International Cryogenic Engineering Conference, Eindhoven. Guilford, UK: IPC Science and Technology Press; 1972. pp. $47-54$ 
[4] Sankha B, Bhupendra P. A review on cryoprotectant and its modern implication in cryonics. Asian Journal of Pharmaceutics. 2016:10(3):10.1-10.6

[5] Pegg DE. Cryobiology-A review. In: Timmerhaus KD, editor. Advances in Cryogenic Engineering. New York, NY: Plenum Publishing Corporation; 1972. pp. 116-136

[6] Osetsky AL. Thermodynamic aspects of cluster crystallization in cryoprotective solution. Cryo Letters. 2011;32(3):216-224

[7] Fahy M. Cryoprotectant toxicity and cryoprotectant toxicity reduction. Cryobiology. 1990;27(3):247-268

[8] Karow AM. Cryoprotectants-A new class of drugs. Journal of Pharmacy and Pharmacology. 1969;21:209-223

[9] Leibo SP. The principle variable of cryopreservation. Fertility and Sterility. 2011;96(2): 269-276

[10] Fahy M, MacFarlane DR, Angell CA, Meryman HT. Vitrification as an approach to cryopreservation. Cryobiology. 1984;21:407-426

[11] Fahy GM. The relevance of cryoprotectant "toxicity" to cryobiology. Cryobiology. 1986; 23:1-13

[12] Fahy GM. Cryoprotectant toxicity and cryoprotectant toxicity reduction: In search of molecular mechanisms. Cryobiology. 1990;27:247-268

[13] Fahy GM, Wowk B, Wu J, Paynter S. Improved vitrification solutions based on the predictability of vitrification solution toxicity. Cryobiology. 2004;48:22-35

[14] Imrat P, Suthanmapinanth P, Saikhun K, Mahasawangkul S, Sostaric E, Sombutputorn $\mathrm{P}$, et al. Effect of pre-freeze semen quality, extender and cryoprotectant on the post-thaw quality of Asian elephant. Cryobiology. 2013;66(1):52-59

[15] Joshi AJ. A review and application of cryoprotectant: The science of cryonics. Pharma Tutor. 2016;4(1):12-18

[16] Wowk B, Fahy GM. Inhibition of bacterial ice nucleation by polyglycerol polymers. Cryobiology. 2002;44(1):14-23

[17] Gndolfi F. Efficiency of equilibrium cooling and rapid freezing procedure for cryopreservation of rat ovarian tissue. Fertility and Sterility. 2006;81(1):1150-1156

[18] Wang X, Chen H, Yin H, Kim S, Lin Tan S, Gosden R. Fertility after intact ovary transplantation. Nature. 2002;415:385

[19] Arav A, Revel A, Nathan Y, Bor A, Gacitua H, Yavin S, et al. Oocyte recovery, embryo development and ovarian function after cryopreservation and transplantation of whole sheep ovary. Human Reproduction. 2005;20:3554-3559

[20] Dittrich R, Maltaris T, Mueller A, Dimmler A, Hoffmann I, Kiesewetter F, et al. Successful uterus cryopreservation in an animal model. Hormone and Metabolic Research. 2006; 38:141-145 
[21] Berejnov V, Husseini NS, Alsaied OA, Thorne RE. Effects of cryoprotectant concentration and cooling rate on vitrification of aqueous solutions. Journal of Applied Crystallography. 2006;39:244-225

[22] Anderson RA, Wallace WH, Baird DT. Ovarian cryopreservation for fertility preservation: Indications and outcomes. Reproduction. 2008;136(6):681-689

[23] Chong YK, Toh TB, Zaiden N, Poonepalli A, Leong SH, Ong CE, et al. Cryopreservation of neurospheres derived from human glioblastoma multiforme. Stem Cells. 2009;27(1):29-39

[24] Cetinkaya G, Arat S. Cryopreservation of cartilage cell and tissue for bio-banking. Transfusion Medicine and Hemotherapy. 2011;38(2):107-123

[25] Wong KM, Mastenbroek S, Repping S. Cryopreservation of human embryos and its contribution to in vitro fertilization success rates. American Society for Reproductive Medicine. 2014;102(1):19-26

[26] Silva AR, Lima GL, Peixoto GCX, Souza ALP. Cryopreservation in mammalian conservation biology: Current applications and potential utility. RRBS. 2015;4:1-8

[27] Voelkel SA, Hu YX. Use of ethylene glycol as a cryoprotectant for bovine embryos allowing direct transfer of frozen-thawed embryos to recipient females. Theriogenology. 1992;37(3):687-697

[28] Pop OL, Diaconeasa Z, Brandau T, Ciuzan O, Pamfil D, Vodnar D, et al. Effect of glycerol, as cryoprotectant in the encapsulation and freeze drying of microspheres containing probiotic cells. Bulletin of University of Agricultural Sciences and Veterinary Medicine Cluj-Napoca. Food Science and Technology. 2015;72. DOI: 10.15835/buasvmcn-fst:10993

[29] Chung N-O, Kyung Lee M, Lee J. Mechanism of freeze-drying drug nanosuspensions. International Journal of Pharmaceutics. 2012;437;42-50. DOI: 10.1016/j.ijpharm.2012. 07.068

[30] Zhao J, Wang Y, Ma Y, Liu Y, Yan B, Wang L. Smart nanocarrier based on PEGylated hyaluronic acid for deacetyl mycoepoxydience: High stability with enhanced bioavailability and efficiency. Carbohydrate Polymers. 2019;203:356-368. DOI: 10.1016/j. carbpol.2018.09.071

[31] Gosden RG, Yin H, Bodine RJ, Morris GJ. Character, distribution and biological implications of ice crystallization in cryopreserved rabbit ovarian tissue revealed by cryoscanning electron microscopy. Human Reproduction. 2010;25(2):470-478 
